\title{
A multi-period dial-a-ride problem with driver consistency
}

\author{
Kris Braekers*†1 and Attila Kovacs ${ }^{2}$ \\ ${ }^{1}$ Hasselt University - Belgium \\ ${ }^{2}$ University of Southampton - United Kingdom
}

\begin{abstract}
Dial-a-ride services provide disabled and elderly people with a personalized mode of transportation to preserve their mobility. Typically, several users with different pickup and drop off locations are transported on a vehicle simultaneously. The focus in Dial-A-Ride Problems is mainly on minimizing routing cost. Service quality has been taken into account by imposing time windows and limiting the maximum ride time of each user. We extend the classical DARP by an additional feature of service quality referred to as driver consistency. Users of dial-a-ride services are often sensitive to changes in their daily routine. This aspect includes the person who is providing the transportation service, i.e., the driver of the vehicle. Our problem, called the Driver Consistent Dial-A-Ride Problem, considers driver consistency by bounding the maximum number of different drivers that transport a user over a multiperiod planning horizon. We propose different formulations of the problem and examine their efficiency when applied in a branch-and-cut fashion. Additionally, we develop a large neighborhood search algorithm that generates near-optimal solutions in a short amount of time. Extensive computational experiments are conducted in order to assess the quality of the solution approaches. Results reveal that the cost of offering driver consistency varies greatly in magnitude. Depending on the instance, the cost of assigning one driver to each user can be up to $22.33 \%$ higher compared to a low-cost solution. However, routing cost increases by not more than $3.75 \%$ if users are transported by at least two drivers.
\end{abstract}

\footnotetext{
*Speaker

${ }^{\dagger}$ Corresponding author: kris.braekers@uhasselt.be
} 